\title{
Effect of Helicobacter pylori infection on the sonic hedgehog signaling pathway in gastric cancer cells
}

\author{
JIE-HYUN KIM ${ }^{1,2^{*}}$, YEUN JUNG CHOI ${ }^{2,4 *}$, SANG HUN LEE ${ }^{2,4}$, HYUN SOO SHIN $^{2}$, IN OHK LEE IN $^{2,4}$

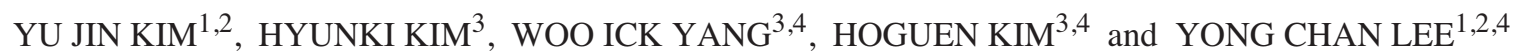 \\ ${ }^{1}$ Department of Internal Medicine, ${ }^{2}$ Institute of Gastroenterology, ${ }^{3}$ Department of Pathology, \\ ${ }^{4}$ Brain Korea 21 Project for Medical Science, Yonsei University College of Medicine, Seoul, Korea
}

Received December 22, 2009; Accepted March 26, 2010

DOI: $10.3892 /$ or_00000791

\begin{abstract}
This study aimed to investigate the effect of Helicobacter pylori (H. pylori) infection on the sonic Hedgehog (Shh) signaling in gastric cancer. Shh, Patched (Ptch), and transcription factor Gli1 were overexpressed in $H$. pyloriinfected gastric cancer cells. The oncoprotein, CagA positive $H$. pylori resulted in significantly higher Shh expression. Pretreatment with MG-132 or PDTC significantly lowered Shh expression. Significant overexpression of Shh and Gli1 were noted in $H$. pylori-infected compared to non-infected gastric cancer tissues. Conclusively, H. pylori activated the Shh signaling pathway in CagA-dependent manner partly through the $\mathrm{NF}-\kappa \mathrm{B}$ pathway in gastric cancer cells.
\end{abstract}

\section{Introduction}

The Hedgehog (Hh) signaling pathway plays a critical role in embryonic development, tissue polarity, and carcinogenesis $(1,2)$. Secreted Hh molecules bind to the Patched (Ptch; Ptch-1, Ptch-2) receptor, alleviating Ptch-mediated suppression of smoothened (Smo), a putative seven-transmembrane protein. Smo signaling triggers a cascade of intracellular events, leading to activation of the pathway through glioma-associated oncogene (Gli)-dependent transcription (2). In the stomach, sonic hedgehog (Shh) is almost exclusively expressed in parietal cells, influencing fundic gland differentiation (3).

Gastric cancer is one of the most common malignancies in the world, and Helicobacter pylori $(H$. pylori) is a major risk factor linked to gastric carcinogenesis. H. pylori may participate in gastric carcinogenesis through the interaction

Correspondence to: Dr Yong Chan Lee, Department of Internal Medicine, Yonsei University College of Medicine, 134 ShinchonDong, Seodaemun-Ku, Seoul 120-752, Korea

E-mail: leeyc@yuhs.ac

*Contributed equally

Key words: gastric cancer, sonic hedgehog, Helicobacter pylori, carcinogenesis between bacterial factors such as CagA and host signal transduction pathways (4). Shh expression is significantly lower in $H$. pylori-associated gastritis or with intestinal metaplasia (IM) changes $(3,5-7)$. This reduction correlates with the loss of parietal cells on the morphogenic bases $(3,5-7)$. However, some studies report overexpression of Shh in human gastric cancers (8-10). Therefore, we examined Shh signaling pathway changes in gastric cancer cells in response to $H$. pylori infection in order to elucidate the influence of Shh signaling activation in $H$. pylori-mediated gastric carcinogenesis.

\section{Materials and methods}

Gastric cancer cells and H. pylori infection. AGS (ATCC CRL 1739), MKN 28 (JCRB 0253), MKN45 (KCLB 80103), and KATO III (KCLB 30103) gastric cancer cells were maintained in RPMI-1640 medium (Gibco, Grand Island, NY) supplemented with $10 \%$ fetal bovine serum (Gibco) and $1 \%$ penicillin-streptomycin sulfate (Gibco). All cultures were maintained in a $37^{\circ} \mathrm{C}$ incubator supplemented with $5 \% \mathrm{CO}_{2}$. $H$. pylori strains were cultured on blood agar plates containing $5 \%$ sheep blood in a $37^{\circ} \mathrm{C}$ incubator with a gas pak (BD, Sparks, MA). We cultured H. pylori strains 8822 (cagAnegative), 60190 (cagA-positive), and $\triangle \mathrm{cagA}$ (isogenic mutant lacking $\operatorname{cagA}$; a kind gift from Professor Richard M. Peek Jr, Vanderbit University). For $H$. pylori infection of gastric cancer cells, $H$. pylori strains were cultured for 24 to $36 \mathrm{~h}$. Cultured $H$. pylori were harvested in phosphate-buffered saline (PBS, pH 7.4) and added to the serum-starved host cells at a multiplicity of infection (MOI) of 100 .

Reagents. The Smo-specific inhibitor cyclopamine (Calbiochem, La Jolla, CA) was dissolved in DMSO. Proteasome inhibitor MG-132 (Sigma-Aldrich, St. Louis, MO) and pyrrolidine dithiocarbamate (PDTC) (Sigma-Aldrich), a specific inhibitor of nuclear factor- $\kappa \mathrm{B}(\mathrm{NF}-\kappa \mathrm{B})$, were used at previously described concentrations (11).

Tissue samples and immunohistochemistry. Surgical specimens were obtained from 20 patients with gastric cancer. Tissue sections in microslides were deparaffinized with xylene, hydrated in serial dilutions of alcohol, and immersed in $3 \%$ $\mathrm{H}_{2} \mathrm{O}_{2}$. Following antigen retrieval in citrate buffer ( $\mathrm{pH} 6.0$ ), the tissue sections were incubated with protein blocking 
(a)

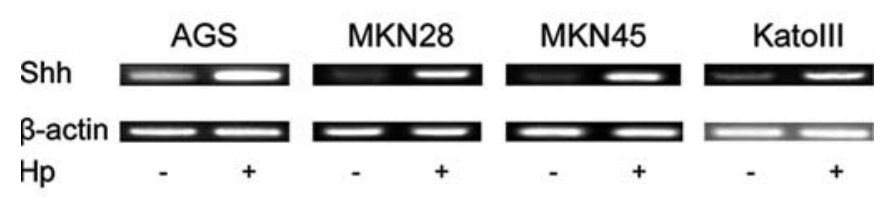

(b)

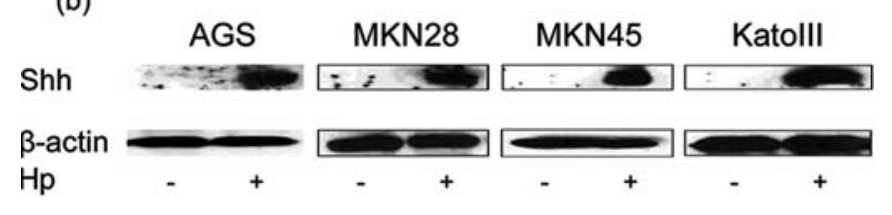

Hp, Helicobacter pylori 60190

Figure 1. Helicobacter pylori (H. pylori) infection induced sonic hedgehog (Shh) overexpression in gastric cancer cells. (a) AGS, MKN-28, MKN-45, and KATO-III cells were infected by cag A-positive H. pylori (60190), and RT-PCR for Shh mRNA was performed. (b) Immunoblot of Shh expression in the gastric cancer cell lines.

agent (Immunotech, Coulter, Inc., Marseille, France) to block nonspecific antibody binding for $20 \mathrm{~min}$ at room temperature and then incubated overnight at $4^{\circ} \mathrm{C}$ with respective primary polyclonal goat antibodies against human SHH (H-160, Santa Cruz, 1:50) and Gli1 (H-300, Santa Cruz, 1:50). After washing with PBS three times, the sections were incubated with a biotinylated secondary antibody (goat anti-rabbit IgG, Immunotech, Coulter, Inc.) and streptavidin conjugated to horseradish peroxidase (Immunotech) for $20 \mathrm{~min}$ at room temperature, followed by a PBS wash. The chromogen was developed for $5 \mathrm{~min}$ with liquid 3,3'-diaminobenzidine (Immunotech). Next, slides were counterstained with Meyer's hematoxylin. Expression status was quantified by scoring both the intensity and proportion of SHH or Gli1 staining. The intensity of cytoplasmic SHH staining was scored as 0, negative (weak or similar to background); 1 , weak (less intense than normal cells); 2 , moderate (similar intensity to normal cells); and 3, strong (stronger than normal cells). The intensity of GLI1 staining was scored as 0 , no detectable nuclear staining; 1, weak nuclear staining; and 2, strong nuclear staining. The proportion of expression in each tissue was determined by counting positively stained cancer cells in relation to a total of three hundred cells, and expressing the result as a percentage.

Real-time reverse transcription-polymerase chain reaction (RT-PCR) analyses. Total RNA was extracted from cultured cells using an RNeasy mini kit (Qiagen, Tokyo, Japan). The RNA was reverse transcribed using oligo (dT) primers and Superscript $^{\mathrm{TM}}$ II reverse transcriptase (Invitrogen, Carlsbad, CA). PCR was performed with a PCR Maxi kit (iNtRON, Sungnam, Korea) according to the manufacturer's instructions. Amplification conditions included denaturation at $95^{\circ} \mathrm{C}$ for $5 \mathrm{~min}$, followed by 30 cycles of $30 \mathrm{sec}$ each at 95,57 , and $72^{\circ} \mathrm{C}$ for Shh, 30 cycles of $30 \mathrm{sec}$ each at 95,57 , and $72^{\circ} \mathrm{C}$ for Ptch1, 30 cycles of $30 \mathrm{sec}$ each at 95,59 , and $72^{\circ} \mathrm{C}$ for Gli1, and 18 cycles of $30 \mathrm{sec}$ each at 95,60 , and $72^{\circ} \mathrm{C}$ for $\beta$-actin. The following primer pairs were used: $\beta$-actin, $5^{\prime}$-TTG CCG ACA GGA TGC AGA AGA-3' and 5'-AGG TGG (a)

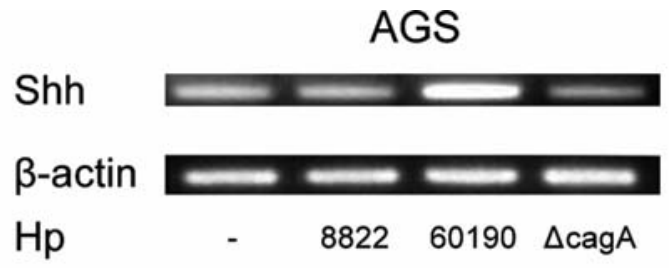

(b)

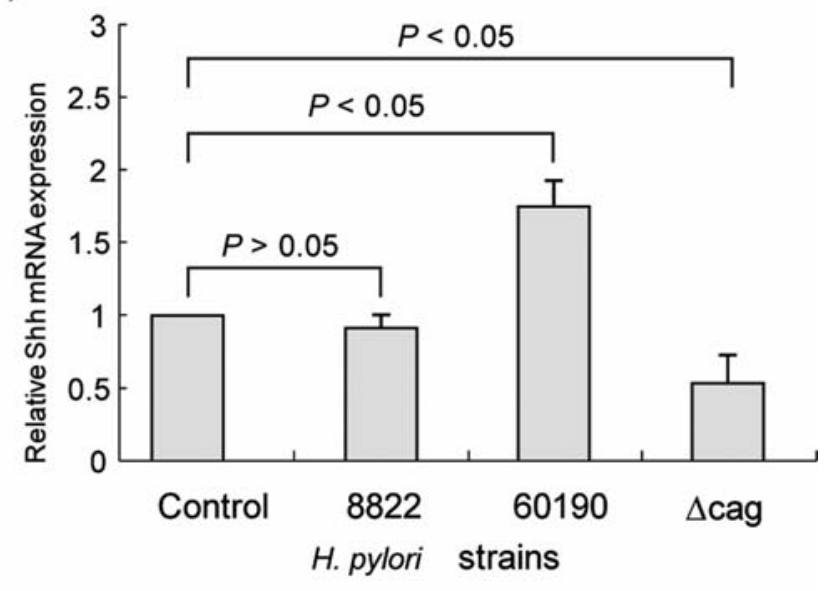

(c)

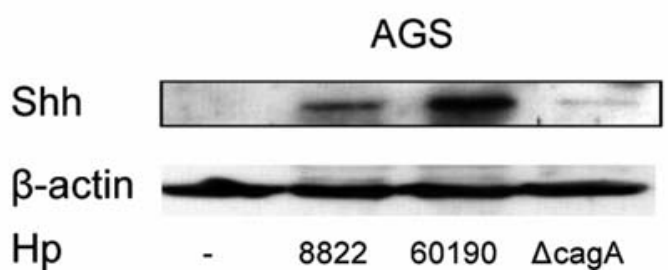

Figure 2. H. pylori infection induced sonic hedgehog (Shh) overexpression in gastric cancer cells in a cagA-dependent manner. (a) AGS cells were infected by cagA-positive (60190) and -negative $(\Delta$ cag, 8822) H. pylori strains, and RT-PCR for Shh mRNA was performed. (b) Shh mRNA expression was also examined by real-time RT-PCR. Relative Shh mRNA levels are shown after normalization to corresponding $\beta$-actin mRNA levels. Columns, mean of three independent experiments; bars, SD. (c) Immunoblot of SHH expression.

ACA GCG AGG CCA GGA T-3'; Shh, 5'-GAG ATG TCT GCT GCT AGT CC-3' and 5'-GTT TCT GGA GAT CTT CCC TT-3'; Ptch1, 5'-CTC CCT ACA GCA GCC ACA GC-3' and 5'-AGG TCC CTT GTG GAG CTG GT-3'; and Gli1 5'-TAT GGA CTT CCC ACC TAC TG-3' and 5'-AAT GTT CAA GTC GAG GAC AC-3'. Real-time RT-PCR was carried out with a PCR mixture containing $1 \mu \mathrm{mol} / 1$ of each primer and SYBR Green master mix (Applied Biosystems, Foster City, CA). The amplifications were conducted at $95^{\circ} \mathrm{C}$ for $10 \mathrm{sec}$ and $60^{\circ} \mathrm{C}$ for $60 \mathrm{sec}$ using an ABI PRISM 7000 Quantitative PCR system (Applied Biosystems). Each sample was examined in triplicate and the amount of PCR product was normalized with respect to $\beta$-actin as an internal control.

Immunoblotting. Prepared cells were harvested after washing with PBS. Collected cells were lysed with buffer [50 mM Tris-Cl (pH 7.5), 150 mM NaCl, 1 mM EDTA (pH 8.0), $1 \%$ Triton X-100, $1 \mathrm{mM}$ PMSF, $1 \mathrm{mM} \mathrm{Na} \mathrm{VO}_{4}$, and protease inhibitor cocktail (Roche, Molecular Biochemical, Indianapolis, 
(a)

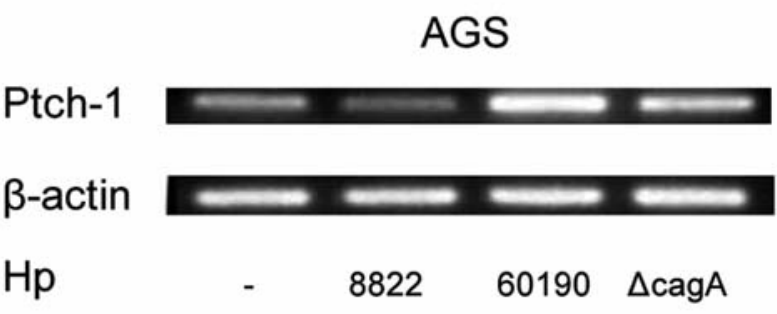

(b)

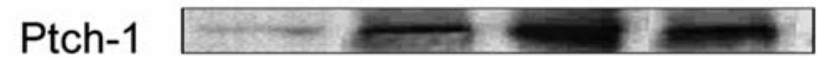

\section{$\beta$-actin}

$\mathrm{Hp}$
Figure 3. H. pylori infection induced patched-1 (Ptch-1) overexpression in gastric cancer cells. (a) RT-PCR analysis of the expression of Ptch-1 in AGS cells. (b) PTCH1 protein as shown by immunoblotting in AGS cells.

IN)]. The same amount of protein was boiled at $95^{\circ} \mathrm{C}$ after adding SDS sample buffer [62.5 mM Tris-Cl (pH 6.8), 2\% sodium dodecyl sulfate, $10 \%$ glycerol, $B$-mercaptoethanol, and $0.002 \%$ bromophenol blue]. Samples were loaded in $12 \%$ SDS-PAGE gels for SHH and $8 \%$ SDS PAGE gels for PTCH1 and GLI1 and then transferred to PVDF membranes (Amersham Biosciences, Pisctaway NJ). Rabbit anti-SHH (Santa Cruz, 1:1000) and anti-GLI1 (Santa Cruz, 1:1000) were used as the primary labeling antibodies and the appropriate horseradish peroxidase-conjugated antibodies (Santa Cruz) were used as secondary antibodies. An enhanced chemiluminescence detection system (ECL-Plus, iNtRON, Seoul, Korea) was used for detection according to the manufacturer's protocol.

Statistical analyses. Statistical analyses were conducted using the Student t-test for real-time RT-PCR and Pearson's Chi-square test for immunohistochemistry. A two-sided p-value of $<0.05$ was considered statistically significant. All statistical analyses were performed using SPSS software (SPSS 12.0, Chicago, IL, USA).

\section{Results}

SHH expression correlated with H. pylori infection in gastric cancer cells. To examine whether $H$. pylori infection was associated with increased expression of Shh mRNA and protein, we performed in vitro experiments with four different types of human gastric cancer cell lines. We first examined whether $H$. pylori infection altered Shh mRNA transcription. We infected the cells with bacterial strains 8822 (cagAnegative), 60190 (cagA-positive), and $\Delta c a g A$ (cagA knockout mutant) for $6 \mathrm{~h}$ in order to evaluate the effect of cagA status on Shh expression by gastric cancer cells. The transcription of Shh mRNA was examined by real-time RTPCR. H. pylori infection increased the transcription of Shh mRNA (Fig. 1a), and infection with strain 60190 enhanced the transcription of Shh mRNA more significantly than strain 8822 or $\triangle \operatorname{cagA}$ (Fig. 2).

To confirm that the Shh production is related to $H$. pylori infection in gastric cancer cells, we performed immunoblotting analyses. In all four cell lines, $H$. pylori infection increased SHH expression (Fig. 1b). Furthermore, infection by the cagA-positive strain 60190 caused higher $\mathrm{SHH}$ expression than infection with cagA-negative 8822 or $\Delta c a g A$ (Fig. 2c) bacteria. These findings suggest that $H$. pylori infection affected SHH mRNA transcription and enhanced Shh protein expression in gastric cancer cells in a cagAdependent manner.

Overexpression of Shh induced by H. pylori led to enhanced Hh pathway activation in gastric cancer cells. We examined ligand-dependent $\mathrm{Hh}$ pathway activation in gastric cancer cells. As described above, we infected gastric cancer cells with $H$. pylori to promote overexpression of Shh, and then monitored expression of Ptch-1 and Gli-1, target genes of the Hh pathway, by RT-PCR and immunoblotting. Cells infected with $H$. pylori expressed higher levels of Ptch-1 and Gli-1 than uninfected controls. Furthermore, the 60190 strain significantly induced a greater magnitude expression of both genes in AGS cells (Figs. 3, and 4a and b). These data suggest that overexpression of Shh enhanced Hh signaling pathway activation in gastric cancer cells in a cagA-dependent manner.

To confirm this finding, we treated AGS cells infected by $H$. pylori with cyclopamine to suppress the Hh signaling pathway. Immunoblotting revealed that cyclopamine significantly suppressed GLI1 protein overexpression induced by H. pylori infection (Fig. 4c). These data further confirm that $H$. pylori-induced Shh expression activated the Hh pathway in gastric cancer cells.

$H$. pylori-induced Shh expression is related to the $N F-\kappa B$ pathway. H. pylori has been shown to induce chemokine expression and NF- $\mathrm{KB}$ activation through the ligation of tolllike receptor (TLR) 2 and TLR 5 (12). We questioned whether H. pylori-induced overexpression of Shh protein could be mediated through the NF-кB pathway. AGS cells were pretreated with a NF-кB inhibitor, MG-132 $(2 \mu \mathrm{M})$, for $1 \mathrm{~h}$ before infection with $H$. pylori. The expression of Shh protein was analyzed by immunoblotting. MG-132 treatment resulted in a near complete inhibition of SHH protein expression by AGS cells (Fig. 5a). These results indicate that $H$. pylori-induced Shh expression in AGS cells is mediated through NF-кB activation. To confirm this result, AGS cells were pretreated with another specific NF-кB inhibitor, PDTC, for $6 \mathrm{~h}$ before $H$. pylori infection. When treated with $H$. pylori in the presence of PDTC, levels of Shh were also significantly lower than in controls (Fig. 5b).

SHH expression correlated positively with $H$. pylori infection in human gastric cancer tissues in vivo. To examine whether $H$. pylori infection was associated with Hh signal expression in gastric cancer in vivo, we immunostained a series of 20 
(a)

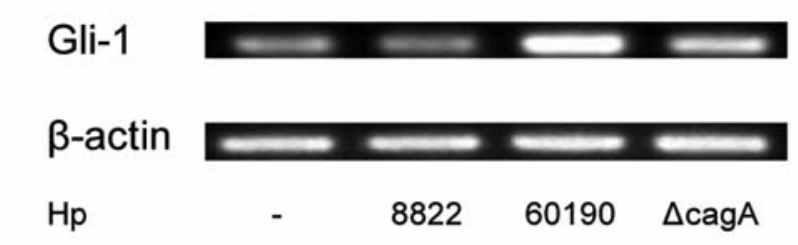

(b)

Gli-1

$\beta$-actin

$\mathrm{Hp}$

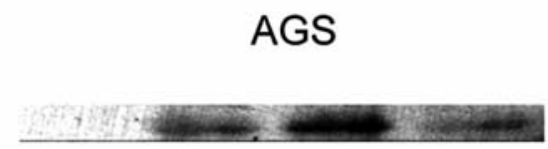

(c)

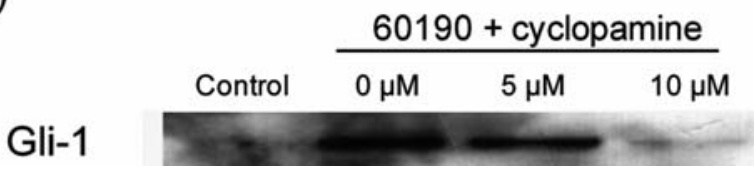

Figure 4. H. pylori infection induced glioma-associated oncogene (Gli)-1 overexpression in gastric cancer cells. (a) RT-PCR analysis of the expression of Gli-1 in AGS cells. (b) GLI1 protein as shown by immunoblotting in AGS cells. (c) AGS cells were infected with $H$. pylori after they were pretreated with cyclopamine for $6 \mathrm{~h}$. Immunoblotting was done with antiGli-1 antibodies.

paraffin-embedded surgical specimens, including 10 specimens of well-differentiated carcinomas $(5 \mathrm{H}$. pylori-positive and $5 \mathrm{H}$. pylori-negative) and 10 specimens of poorlydifferentiated carcinomas $(5 \mathrm{H}$. pylori-positive and $5 \mathrm{H}$. pylori-negative). Expression of SHH and GLI1 proteins was analyzed in each specimen. SHH expression was mainly detected in the cytoplasm (Fig. 6) and GLI1 proteins were strongly expressed in both the cytoplasm and nuclei of gastric cancer cells (Fig. 7). H. pylori-positive samples displayed higher $\mathrm{SHH}$ staining ratios than the H. pylorinegative samples in each type of differentiated tissue $(\mathrm{p}<0.05)$ (Table I). The percentage of cells with nuclear staining of GLI1 showed a similar pattern $(\mathrm{p}<0.05)$ (Table II). These data suggest that $H$. pylori infection was associated with overexpression of $\mathrm{SHH}$, resulting in activation of Hh signaling pathways in gastric cancer tissues in vivo.

\section{Discussion}

The evolution of $H$. pylori-associated gastric cancer begins with superficial gastritis that progresses to chronic gastritis, atrophy with islands of IM, development of dysplasia, and finally frank carcinoma $(13,14)$. Since $\mathrm{SHH}$ is one of the peptide morphogens expressed in the parietal cells of normal human stomachs, the loss of fundic glands due to $H$. pylori-related atrophic changes or IM is accompanied by the loss of Shh expression $(5,15,16)$. Thus, loss of Shh expression due to $H$. pylori may be an early change that occurs in the mucosa (a)

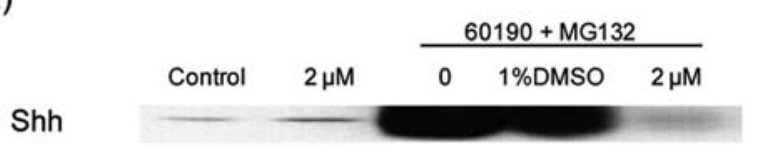

(b)

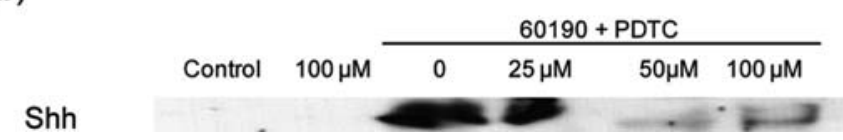

Figure 5. H. pylori induced sonic hedgehog ( $\mathrm{SHH}$ ) protein expression through the NF- $\mathrm{KB}$ pathway in AGS cells. (a) AGS cells were infected with H. pylori after they were pretreated with MG-132 at indicated doses for $1 \mathrm{~h}$. Immunoblotting was done with anti-SHH antibodies. (b) AGS cells were infected with $H$. pylori after they were pretreated with pyrrolidine dithiocarbamate (PDTC) at indicated doses for $6 \mathrm{~h}$. Immunoblotting was done with anti-SHH antibodies.

Table I. Relationship between sonic hedgehog (SHH) expression and Helicobacter pylori positivity.

\begin{tabular}{lrrccc}
\hline & \multicolumn{5}{c}{ Expression intensity of SHH (no., \%) } \\
\cline { 2 - 6 } & \multicolumn{1}{c}{0} & 1 & 2 & 3 & p-value \\
\hline WD Hp $^{+}$ & 11.0 & 6.4 & 23.8 & 58.8 & $<0.05$ \\
WD Hp $^{-}$ & 20.0 & 7.4 & 40.6 & 32.0 & \\
PD Hp $^{+}$ & 0.0 & 31.6 & 50.4 & 18.0 & $<0.05$ \\
PD Hp $^{-}$ & 20.0 & 22.0 & 38.6 & 19.4 & \\
\hline
\end{tabular}

WD, well-differentiated; $\mathrm{PD}$, poorly-differentiated; $\mathrm{Hp}^{+}$, Helicobacter pylori-positive; $\mathrm{Hp}^{-}$, Helicobacter pylori-negative.

Table II. Relationship between glioma-associated oncogene (GLI1) expression and Helicobacter pylori positivity.

\begin{tabular}{lcccc}
\hline & \multicolumn{4}{c}{ Expression intensity of GLI1 (no., \%) } \\
\cline { 2 - 5 } & 0 & 1 & 2 & p-value \\
\hline WD Hp $^{+}$ & 30.8 & 43.5 & 25.7 & $<0.05$ \\
WD Hp $^{-}$ & 51.5 & 35.2 & 13.3 & \\
PD Hp $^{+}$ & 53.2 & 32.5 & 14.3 & $<0.05$ \\
PD Hp $^{-}$ & 57.2 & 33.1 & 9.7 & \\
\hline
\end{tabular}

WD, well-differentiated; $\mathrm{PD}$, poorly-differentiated; $\mathrm{Hp}^{+}$, Helicobacter pylori-positive; $\mathrm{Hp}^{-}$, Helicobacter pylori-negative.

prior to neoplastic transformation. However, the level of Shh is also reportedly elevated in human gastric cancers, especially intestinal types of gastric cancer, which develop through a sequential precancerous cascade of chronic superficial gastritis, chronic atrophic gastritis, IM, and dysplasia (8-10). The discordance of Shh expression levels between preneoplastic lesions and gastric cancer may be a consequence of dual roles 


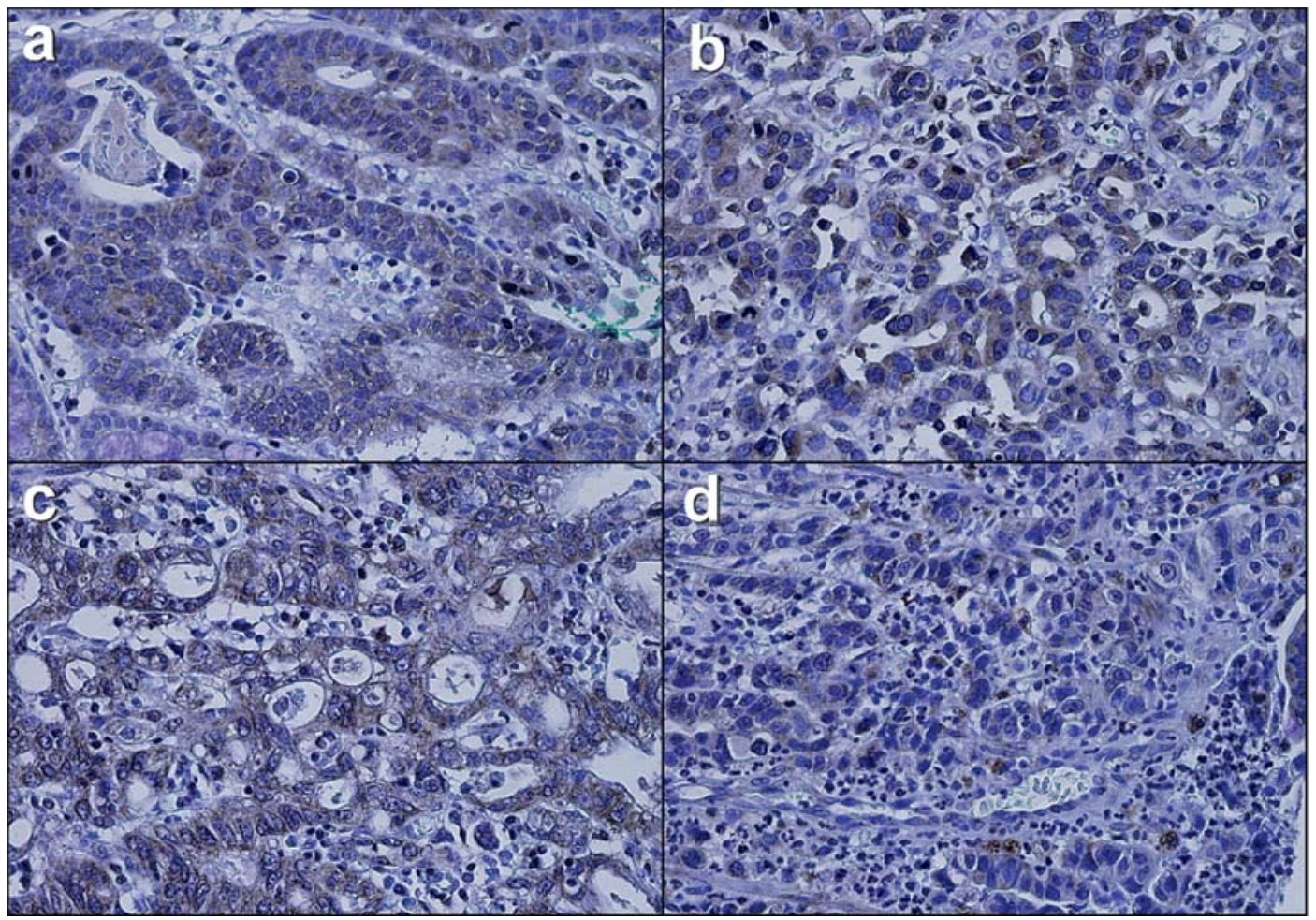

Figure 6. Expression of sonic hedgehog (SHH) in gastric carcinoma tissues. SHH expression was mainly seen in the cytoplasm. SHH staining revealed strong expression in $H$. pylori-positive tissues in each type of differentiated adenocarcinoma (immunohistochemical staining x 400). (a) Well-differentiated $H$. pyloripositive tissue. (b) Well-differentiated H. pylori-negative tissue. (c) Poorly-differentiated H. pylori-positive tissue. (d) Poorly-differentiated $H$. pylori-negative tissue.

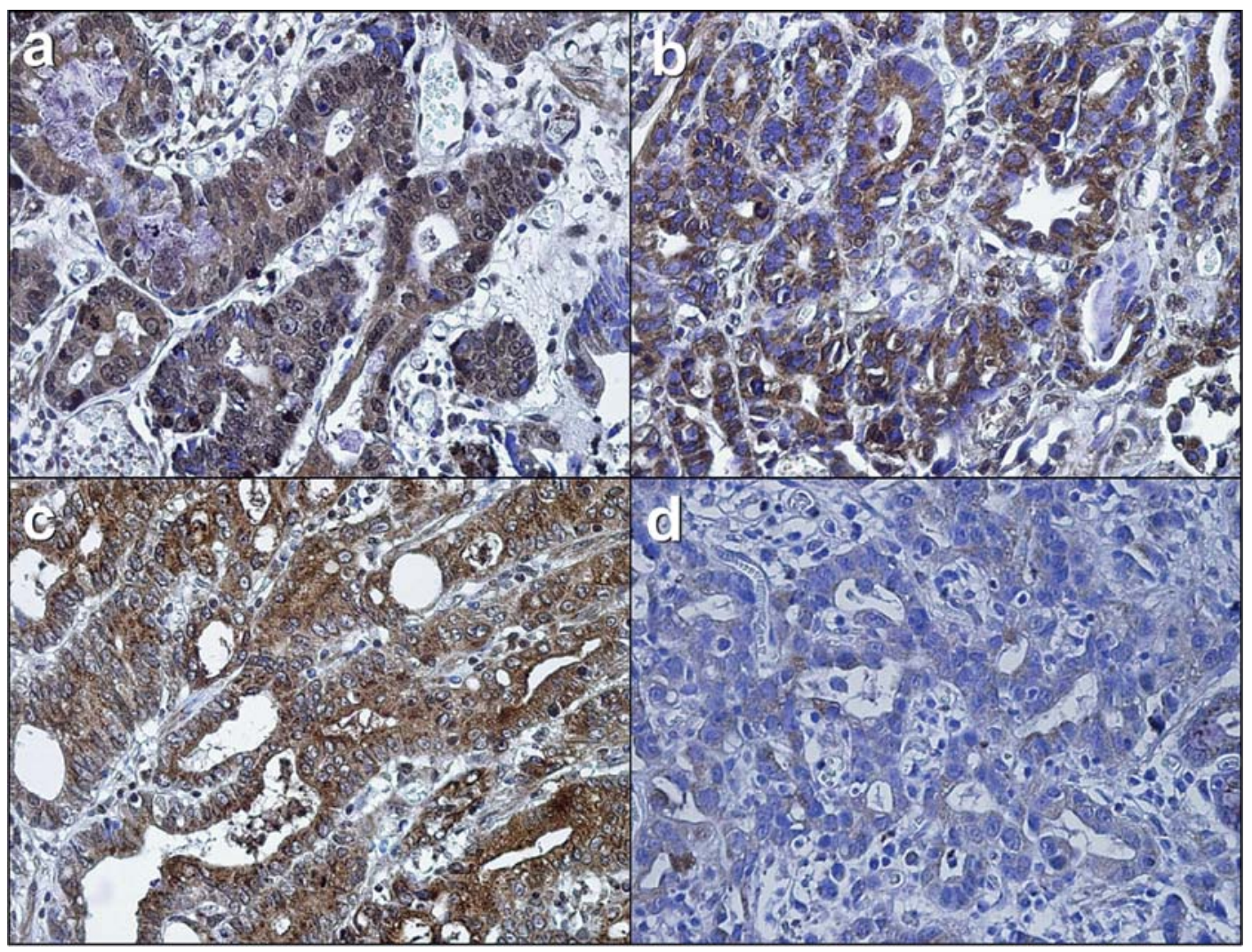

Figure 7. Expression of glioma-associated oncogene (GLI)-1 in gastric carcinoma tissues. The GLI1 protein was expressed in both the cytoplasm and nuclei of gastric carcinoma tissues. GLI1 staining revealed strong expression in $H$. pylori-positive tissues in each type of differentiated adenocarcinoma (immunohistochemical staining x400). (a) Well-differentiated $H$. pylori-positive tissue. (b) Well-differentiated $H$. pylori-negative tissue. (c) Poorly-differentiated H. pylori-positive tissue. (d) Poorly-differentiated H. pylori-negative tissue. 
of Shh in morphogenesis and carcinogenesis. Accordingly, we examined Shh changes following $H$. pylori infection in gastric cancer cells to clarify the role of Hh signal activation in $H$. pylori-associated gastric carcinogenesis.

In the present study, H. pylori activated the Hh signaling pathway through the up-regulation of Shh expression in gastric cancer cells. This Shh overexpression was dependent on cagA, the pivotal virulence factor for gastric carcinogenesis in $H$. pylori infection. This finding suggests that the $\mathrm{Hh}$ signaling pathway is one of the host signal transduction pathways related to gastric carcinogenesis by $H$. pylori. Furthermore, Shh was detected in gastric cancer cells and overexpressed following $H$. pylori infection in gastric cancer cells. These findings suggest that $H$. pylori may be related to cancer growth/metastasis after cancer transformation by overexpression of Shh.

We further found that the Shh overexpression following $H$. pylori infection was mediated through the NF-кB pathway. $\mathrm{NF}-\kappa \mathrm{B}$ is a transcription factor that controls expression of numerous genes involved in inflammation and immune response processes, including proliferation, invasion and adhesion, angiogenesis, and apoptosis (17). More importantly, $H$. pylori induces chemokine expression and NF- $\mathrm{KB}$ activation through the ligation of TLR 2 and TLR 5 receptors (12). We blocked the NF- $\mathrm{KB}$ signal pathway using a specific inhibitor to evaluate whether NF- $\mathrm{KB}$ participates in Shh overexpression by $H$. pylori infection. Blockade of the $N F-\kappa B$ pathway suppressed constitutive Shh expression, in agreement with previous results in pancreatic cancer (18). These findings suggest a possible role of NF- $\mathrm{KB}$ inhibitors in the prevention of $H$. pylori-mediated gastric carcinogenesis. Also, as cagApositive strains were associated with higher levels of Shh expression, our data raise the possibility that intracellularly translocated oncoprotein CagA may be implicated in Shh overexpression through the NF- $\mathrm{KB}$ pathway in gastric cancer cells.

Changes in the level of Shh expression in the gastric epithelium may provide a marker for chemopreventive measures. When the level of Shh declines in H. pyloriassociated gastritis or IM, the interaction between Shh and $H$. pylori is based on the process of morphogenesis in the stomach. Eradication of $H$. pylori, resulting in the restoration of SHH protein expression, can lead to a morphogenetic drive in gastric epithelial homeostasis. However, if the level of Shh is higher in $H$. pylori-associated preneoplastic tissues or gastric cancer during follow-up, the interaction between Shh and H. pylori is converted into a process of cancer progression. Thus, the Hh signaling pathway may play a role in neoplastic transformation and progression in gastric carcinogenesis by $H$. pylori. This stage may be a sign of another "point of no return' in gastric carcinogenesis caused by H. pylori. However, this hypothesis must be further examined by serial experiments involving the $H$. pylori-related precancerous cascade.

In conclusion, $H$. pylori infection activates the $\mathrm{Hh}$ signaling pathway through the up-regulation of Shh in gastric cancer cells. Shh activation is cagA-dependent and mediated through the NF- $\kappa$ B pathway. Accordingly, the Shh signaling pathway should be considered as a possible explanation for $H$. pylorirelated gastric carcinogenesis.

\section{Acknowledgements}

This work was supported by a Korea Research Foundation Grant funded by the Korean Government (MOEHRD) (KRF2006-312-E00105).

\section{References}

1. Pasca di Magliano M and Hebrok M: Hedgehog signalling in cancer formation and maintenance. Nat Rev Cancer 3: 903-911, 2003.

2. Taipale $\mathrm{J}$ and Beachy PA: The Hedgehog and Wnt signalling pathways in cancer. Nature 411: 349-354, 2001.

3. Faller $\mathrm{G}$ and Kirchner T: Immunological and morphogenic basis of gastric mucosa atrophy and metaplasia. Virchows Arch 446: $1-9,2005$

4. Maeda S and Mentis AF: Pathogenesis of Helicobacter pylori infection. Helicobacter 1: 10-14, 2007.

5. Shiotani A, Iishi H, Uedo N, et al: Evidence that loss of sonic hedgehog is an indicator of Helicobater pylori-induced atrophic gastritis progressing to gastric cancer. Am J Gastroenterol 100: 581-587, 2005 .

6. Shiotani A, Uedo N, Iishi H, et al: Re-expression of sonic hedgehog and reduction of CDX2 after Helicobacter pylori eradication prior to incomplete intestinal metaplasia. Int $\mathbf{J}$ Cancer 121: 1182-1189, 2007.

7. Shiotani A, Iishi H, Uedo N, et al: Helicobacter pylori-induced atrophic gastritis progressing to gastric cancer exhibits sonic hedgehog loss and aberrant CDX2 expression. Aliment Pharmacol Ther 24 (Suppl 4): 71-80, 2006.

8. Wang LH, Choi YL, Hua XY, et al: Increased expression of sonic hedgehog and altered methylation of its promoter region in gastric cancer and its related lesions. Mod Pathol 19: 675-683, 2006.

9. Fukaya $\mathrm{M}$, Isohata $\mathrm{N}$, Ohta $\mathrm{H}$, et al: Hedgehog signal activation in gastric pit cell and in diffuse-type gastric cancer. Gastroenterology 131: 14-29, 2006.

10. Lee SY, Han HS, Lee KY, et al: Sonic hedgehog expression in gastric cancer and gastric adenoma. Oncol Rep 17: 1051-1055, 2007.

11. Li Q, Yu YY, Zhu ZG, et al: Effect of NF-kappaB constitutive activation on proliferation and apoptosis of gastric cancer cell lines. Eur Surg Res 37: 105-110, 2005.

12. Smith MF Jr, Novotny J, Carl VS and Comeau LD: Helicobacter pylori and toll-like receptor agonists induce syndecan-4 expression in an NF-kappaB-dependent manner. Glycobiology 16: 221-229, 2006.

13. Dixon MF: Prospects for intervention in gastric carcinogenesis: reversibility of gastric atrophy and intestinal metaplasia. Gut 49: 2-4, 2001.

14. Meining A, Morgner A, Miehlke S, Bayerdorffer E and Stolte M: Atrophy-metaplasia-dysplasia-carcinoma sequence in the stomach: a reality or merely an hypothesis? Best Pract Res Clin Gastroenterol 15: 983-998, 2001.

15. Van den Brink GR, Hardwick JC, Nielsen C, et al: Sonic hedgehog expression correlates with fundic gland differentiation in the adult gastrointestinal tract. Gut 51: 628-633, 2002.

16. Suzuki H, Minegishi Y, Nomoto Y, et al: Down-regulation of a morphogen (sonic hedgehog) gradient in the gastric epithelium of Helicobacter pylori-infected Mongolian gerbils. J Pathol 206: 186-197, 2005

17. Karin M and Greten FR: NF-kappaB: linking inflammation and immunity to cancer development and progression. Nat Rev Immunol 5: 749-759, 2005.

18. Nakashima H, Nakamura M, Yamaguchi $\mathrm{H}$, et al: Nuclear factor-kappaB contributes to hedgehog signaling pathway activation through sonic hedgehog induction in pancreatic cancer. Cancer Res 66: 7041-7049, 2006. 